\title{
NOUVELLE
}

\section{Facteurs neurotrophiques pour les motoneurones}

\section{Un menu à la carte}

Alysson Alory ${ }^{1}$, Arnaud Jacquier ${ }^{1}$, David Gentien², Pierre de la Grange ${ }^{3}$, Georg Haase ${ }^{1}$

\author{
${ }^{1}$ Institut de neurosciences de la Timone, UMR 7289 CNRS et \\ Aix-Marseille Université, 27, boulevard Jean Moulin, \\ 13005 Marseille, France. \\ ${ }^{2}$ Institut Curie, Université PSL, Département de recherche \\ translationnelle, Plateforme de génomique, 26, rue d'Ulm, \\ 75248 Paris, France. \\ ${ }^{3}$ GenoSplice, iPEPS - ICM, Hôpital Pitié Salpêtrière, \\ 47, boulevard de l'Hôpital, 75013 Paris, France. \\ georg.haase@univ-amu.fr
}

> Au cours du développement embryonnaire, environ la moitié des neurones intialement générés sont éliminés. La théorie neurotrophique stipule que la survie ou la mort cellulaire des neurones dépend de leur compétition pour des quantités limitées de facteurs neurotrophiques produits par leurs cibles d'innervation (Figure 1A) [1]. Dans la moelle épinière de l'embryon de poulet par exemple, l'ablation expérimentale d'une ébauche de la patte exacerbe la mort cellulaire des motoneurones. Inversement, la transplantation d'une ébauche de la patte la diminue [1]. À ce jour, plus de quinze facteurs neurotrophiques qui induisent la survie des motoneurones de la moelle épinière ont été décrits [2, 3]. Des études récentes suggèrent que les facteurs neurotrophiques agissent de manière combinatoire $[2,3]$. Chez la souris, l'invalidation génique d'un seul facteur neurotrophique, ou de son récepteur, ne provoque, dans la plupart des cas, qu'une perte modérée des motoneurones [2] tandis que celle de deux ou trois facteurs ou de leurs récepteurs l'accentue. En outre, l'élimination par manipulations génétiques de types cellulaires produisant plusieurs facteurs se traduit par la perte quasi totale des motoneurones [2, 3]. Finalement, certaines combinaisons de facteurs neurotrophiques exercent des effets accrus sur la survie des motoneurones après une axotomie ${ }^{1}$ ou dans des modèles de dégénérescence progressive [4].

\footnotetext{
${ }^{1}$ Une axotomie : section chirurgicale d'un axone.
}

Une hypothèse permettant d'expliquer ces effets combinatoires repose sur l'existence de sous-populations de motoneurones qui présenteraient des besoins trophiques distincts $[2,3,5]$. Cependant, les études cherchant à évaluer cette hypothèse se sont heurtées à plusieurs obstacles: le grand nombre de facteurs neurotrophiques potentiels, la létalité souvent précoce des souris invalidées génétiquement pour ces facteurs ou leurs récepteurs, et l'absence de standardisation des cultures conventionnelles de motoneurones. Pour contourner ces difficultés, nous avons développé une nouvelle approche [6]. Elle consiste à isoler par tri cellulaire (fluorescent-activated cell sorting, FACS), à partir de la moelle épinière lombaire de souris, des motoneurones purs, parfaitement standardisés et hautement viables en culture (Figure $1 B-\varepsilon$ ).

Afin d'étudier les effets combinatoires des facteurs neurotrophiques au cours du développement, douze facteurs, appartenant à des familles différentes de protéines [2], ont été sélectionnés: les neurotrophines BDNF (brain-derived neurotrophic factor) et NT3 (neurotrophine-3); les membres de la famille du GDNF (glial cell line-derived neurotrophic factor), dont le GDNF, la neurturine, l'artémine (ARTN) et la perséphine; les cytokines CNTF (ciliary neurotrophic factor), CTl (cardiotrophine 1) et LIF (leukemia inhibitory factor); et les facteurs de croissance des hépatocytes (HGF, hepatocyte growth factor), et de I'endothélium vasculaire (VEGF, vascular endothelial growth factor), et I'insulin growth factor-1 (IGF-1). Individuellement, chacun de ces facteurs a permis d'améliorer significativement la survie des motoneurones lombaires après trois jours de traitement en culture in vitro (Figure IF), les facteurs les plus efficaces étant le BDNF et le GDNF (Figure IF). Le criblage de 66 combinaisons (ou paires) réalisées à partir de ces douze facteurs a révélé, par la suite, une amplification des effets lorsque trois d'entre eux étaient associés: le HGF, I'ARTN et le CNTF (Figure IF). La triple combinaison de ces trois facteurs était, a fortiori, plus efficace que des combinaisons de paires, permettant le maintien en vie de $46 \%$ des motoneurones (Figure IG). À noter que ce pourcentage équivaut à la somme des effets individuels de chacun des facteurs (Figure IG). Cette stricte additivité suggère donc que les facteurs HGF, ARTN et CNTF, agissent sur des sous-populations distinctes de motoneurones.

\section{Effets distincts des facteurs HGF, CNTF} et ARTN sur des sous-populations de motoneurones

La moelle épinière lombaire est constituée de trois principales sous-populations de motoneurones qui se distinguent par leur position et leurs cibles: les motoneurones de la colonne motrice médiane (MMC) qui innervent les muscles axiaux ; ceux de la colonne motrice latérale (LMC) qui innervent les muscles des membres; et les neurones pré-ganglionnaires (PGC) qui assurent l'innervation sympathique et parasympathique des muscles des sphincters pelviens. 
A
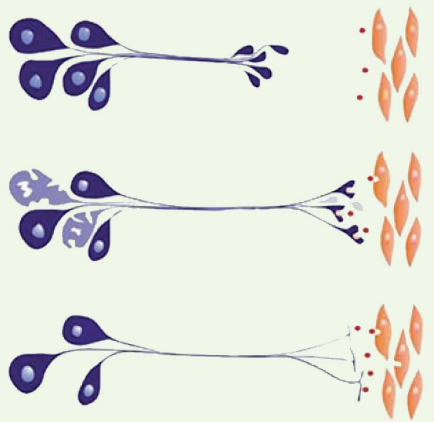

B

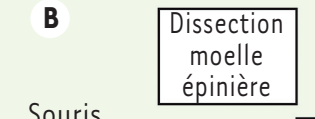
Hb9::GFP
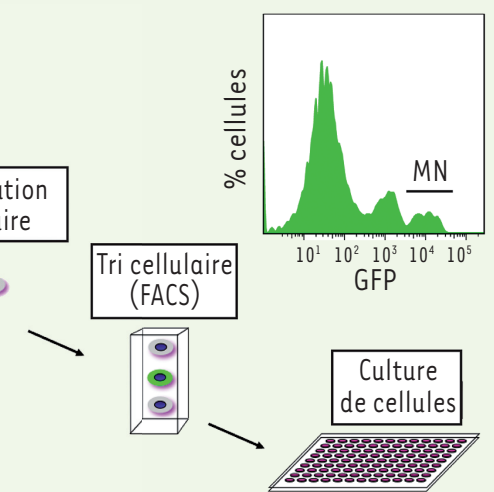

c
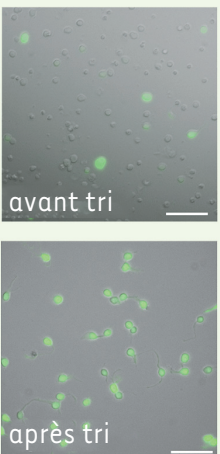

D
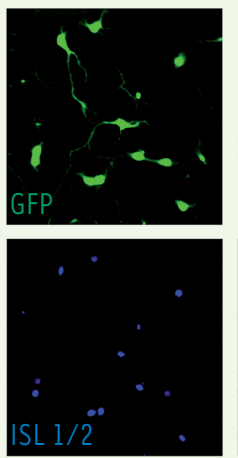
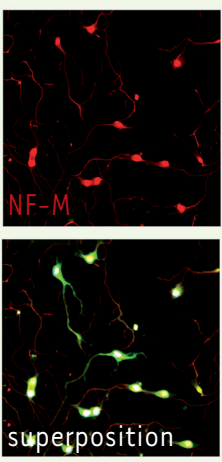

$\varepsilon$

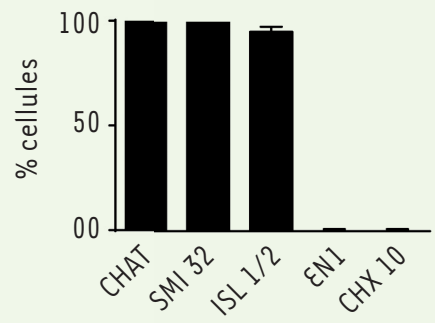

F

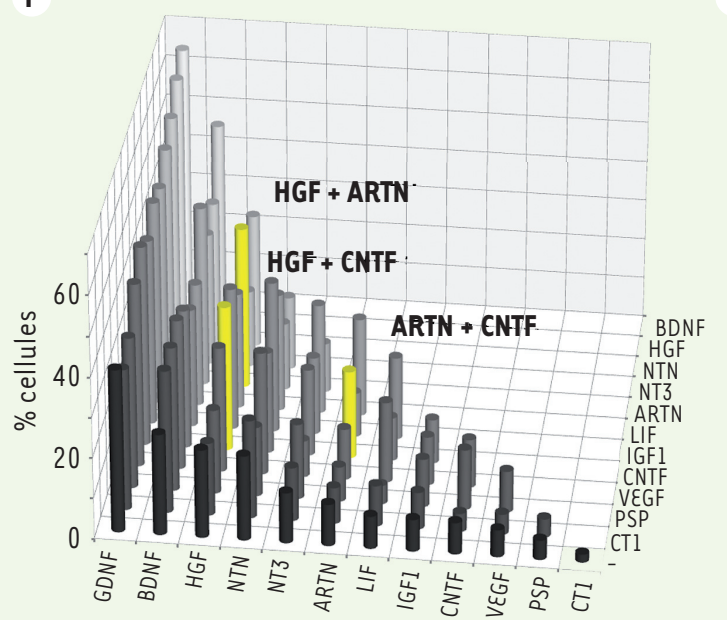

G

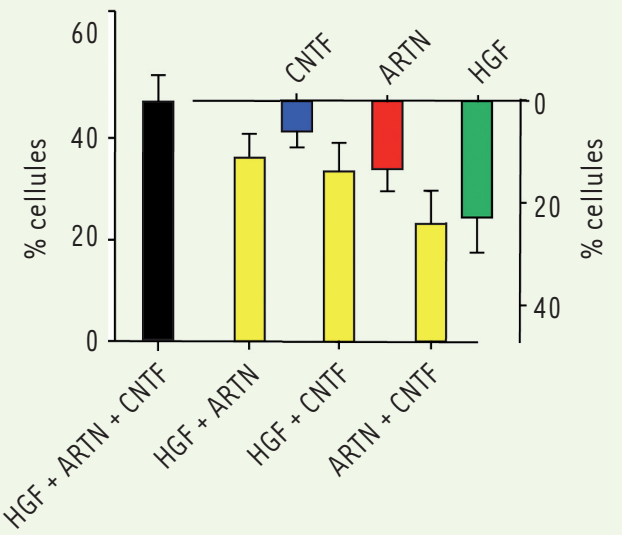

Afin d'étudier la réponse trophique de ces sous-populations, les motoneurones $M M C$ et $L M C / P G C$ ont été isolés par tri cellulaire puis cultivés séparément en présence des facteurs (Figure 2A). Les courbes de survie des cellules après traitement (Figure $2 B$ ), montrent que les facteurs HGF et ARTN favorisent la survie d'une grande proportion de motoneurones LMC/PGC mais qu'ils n'ont qu'un effet mineur sur les moto- neurones MMC. À l'inverse, le CNTF agit spécifiquement sur la survie des motoneurones MMC.

Ces observations ont conduit à étudier l'expression par les motoneurones $M M C$ et $L M C / P G C$, des récepteurs pour les facteurs HGF, CNTF et ARTN, par western blot et l'utilisation de puces à ADN (microarray en anglais) [6]). Dans les motoneurones LMC/PGC, la quantité de la protéine c-Met, le récepteur de HGF, est 4,4 fois plus importante que dans les motoneurones MMC [6], ce qui explique la réponse préférentielle des motoneurones $L M C / P G C$ au traitement par l'HGF. Les composants du récepteur de l'artémine (ARTN), impliquant GFR $\alpha 3$ (GDNF family receptor alpha-3), la tyrosine kinase c-Ret et le syndecan-3 (un protéoglycane de surface), sont également fortement augmentés dans les motoneurones LMC/PGC en accord avec leur 
Figure 1. Effets additifs des facteurs neurotrophiques HGF, CNTF et ARTN sur les motoneurones. A. Schématisation de la compétition de motoneurones embryonnaires (en bleu) pour les facteurs neurotrophiques (en rouge) produits par leurs cibles d'innervation, les cellules musculaires (en orange), selon la théorie neurotrophique. Les motoneurones qui reçoivent suffisamment de facteurs neurotrophiques survivent (en bleu) et les autres sont éliminés (en violet clair) (illustration reproduite avec l'aimable autorisation du Professeur Georg Dechant, université d'Innsbruck, Autriche). B. Isolement de motoneurones. Les prélèvements sont réalisés sur des embryons de souris transgéniques qui expriment la GFP (green fluorescent protein) sous le contrôle du promoteur $\mathrm{Hb} 9$ codant un facteur de transcription à « homéodomaine ». Les motoneurones lombaires qui expriment la GFP, sont isolés par tri cellulaire fluorescent (FACS, fluorescent-activated cell sorting) avant leur mise en culture. Le niveau d'expression de la GFP dans les cellules est représenté par un histogramme indiquant le pourcentage de cellules (en ordonnée) en fonction de l'intensité de fluorescence (en abcisse). Les motoneurones apparaissent à droite (MN), les interneurones au centre et les cellules faiblement fluorescentes à gauche. C. Image de microscopie montrant l'enrichissement en motoneurones, pratiquement à l'homogénéité, après l'étape de tri cellulaire. Barre d'échelle $50 \mu \mathrm{m}$. D. Immunomarquage des motoneurones purifiés et détection de la GFP (en vert), du neurofilament-M (NF-M, en rouge) et du facteur de transcription à homéodomaine ISL $1 / 2$ (en bleu). Ce dernier atteste de la pureté des motoneurones. $\varepsilon$. Les motoneurones isolés sont positifs pour les trois marqueurs spécifiques: CHAT (choline acetyl transférase), SMI 32 (neurofilament-H non phosphorylé) et ISL 1/2, mais négatifs pour les protéines spécifiques d'interneurones comme les facteurs de transcription EN1 (engrailed-1) et CHX 10 (ou VSX2, visual system homeobox 2). F. Criblage des effets de 66 combinaisons de 12 facteurs neurotrophiques sur la survie des motoneurones lombaires. Le diagramme montre les effets de chaque facteur individuel (première rangée de colonnes) et de toutes les combinaisons de facteurs par paires. Les colonnes jaunes indiquent les effets potentialisés des combinaisons (HGF et ARTN), (HGF et CNTF) et (ARTN et CNTF). Le taux de survie est exprimé par rapport aux valeurs obtenues en présence des 12 facteurs réunis (100\%) ou en leur absence ( $0 \%$ ). G. Effets additifs sur la survie des motoneurones des facteurs HGF, ARTN et CNTF combinés par deux ou par trois. HGF : hepatocyte growth factor; ARTN : artémine; CNTF : ciliary neurotrophic factor.

réponse préférentielle à ce facteur. Dans les motoneurones MMC en revanche, I'expression de LIFR $\beta$ (LIF receptor- $\beta$ ) et IL-6st (interleukin 6 signal transducer), deux constituants du récepteur pour le facteur CNTF, est significativement accrue [6], ce qui est en accord avec la réponse spécifique de ces motoneurones à ce facteur. Les neuf autres facteurs neurotrophiques ont des effets similaires sur les différents motoneurones $L M C / P G C$ et $M M C$ et aucune régulation différentielle des récepteurs n'est observée [6].

\section{L'HGF favorise la survie}

des motoneurones LMC innervant les membres

L'implication de ces différents récepteurs dans la survie spécifique des motoneurones a été examinée in vivo, par l'analyse de leur expression et leur localisation, et in vitro, en inhibant dans des cultures cellulaires, la signalisation dont ils sont à l'origine. Les expériences d'hybridation et d'immunolocalisation in situ indiquent que les motoneurones qui expriment le récepteur c-Met forment des groupes de cellules bien délimités localisés dans la colonne motrice latérale (LMC) de la moelle épinière lombaire (Figure 2D). Les expériences d'inhibition in vitro montrent quant à elles, que la réponse de survie à l'HGF peut être complètement abrogée par les inhibiteurs spécifiques de la tyrosine kinase c-Ret ou par des anticorps neutralisant c-Met [6]. L'HGF active donc son récepteur c-Met et favorise, ainsi, la survie d'une fraction de motoneurones de la colonne motrice latérale (LMC) qui innervent les membres.

\section{Le CNTF cible deux sous-populations de motoneurones MMC innervant les muscles axiaux}

Les motoneurones exprimant LIFR $\beta$, élément du récepteur de CNTF, forment deux sous-populations distinctes dans la colonne motrice médiane (MMC) (Figure 2D). Leur survie peut être complètement inhibée par la phosphoinositol phospholipase C (PIPLC) dont l'action induit le détachement de CNTFR $\alpha$ (CNTFreceptor $-\alpha$ ) de la membrane plasmique [6]. Le CNTF favorise donc spécifiquement la survie de motoneurones MMC en activant une signalisation initiée par le CNTFR $\alpha$ et probablement relayée par les récepteurs LIFR $\beta$ et IL6st.

\section{L'ARTN agit comme un facteur} de survie pour les motoneurones préganglionnaires parasympathiques Finalement, nous avons montré que les motoneurones qui expriment le récepteur GFR $\alpha 3$ forment une sous-population distincte (Figure 2D) des autres motoneurones qui présentent les récepteurs c-Met ou LIFR $\beta$. Les marquages rétrogrades réalisés à partir de la vessie [6], suggèrent que cette sous-population distincte correspond aux motoneurones préganglionnaires (PGC) du système nerveux parasympathique. Leur réponse à I'ARTN, en culture, est complètement inhibée par la neutralisation du récepteur GFR $\alpha 3$ ou par l'inhibition du syndecan-3 [6]. Ces résultats identifient I'ARTN comme un facteur de survie des motoneurones préganglionnaires du système nerveux parasympathique.

\section{Conclusion et perspectives}

Notre étude révèle donc que les trois facteurs neurotrophiques, l'HGF, le CNTF et I'ARTN, agissent « à la carte » pour la survie de motoneurones distincts (Figure 2D). L'HGF favorise la survie des motoneurones c-Met de la colonne motrice latérale, ce qui est en parfait 


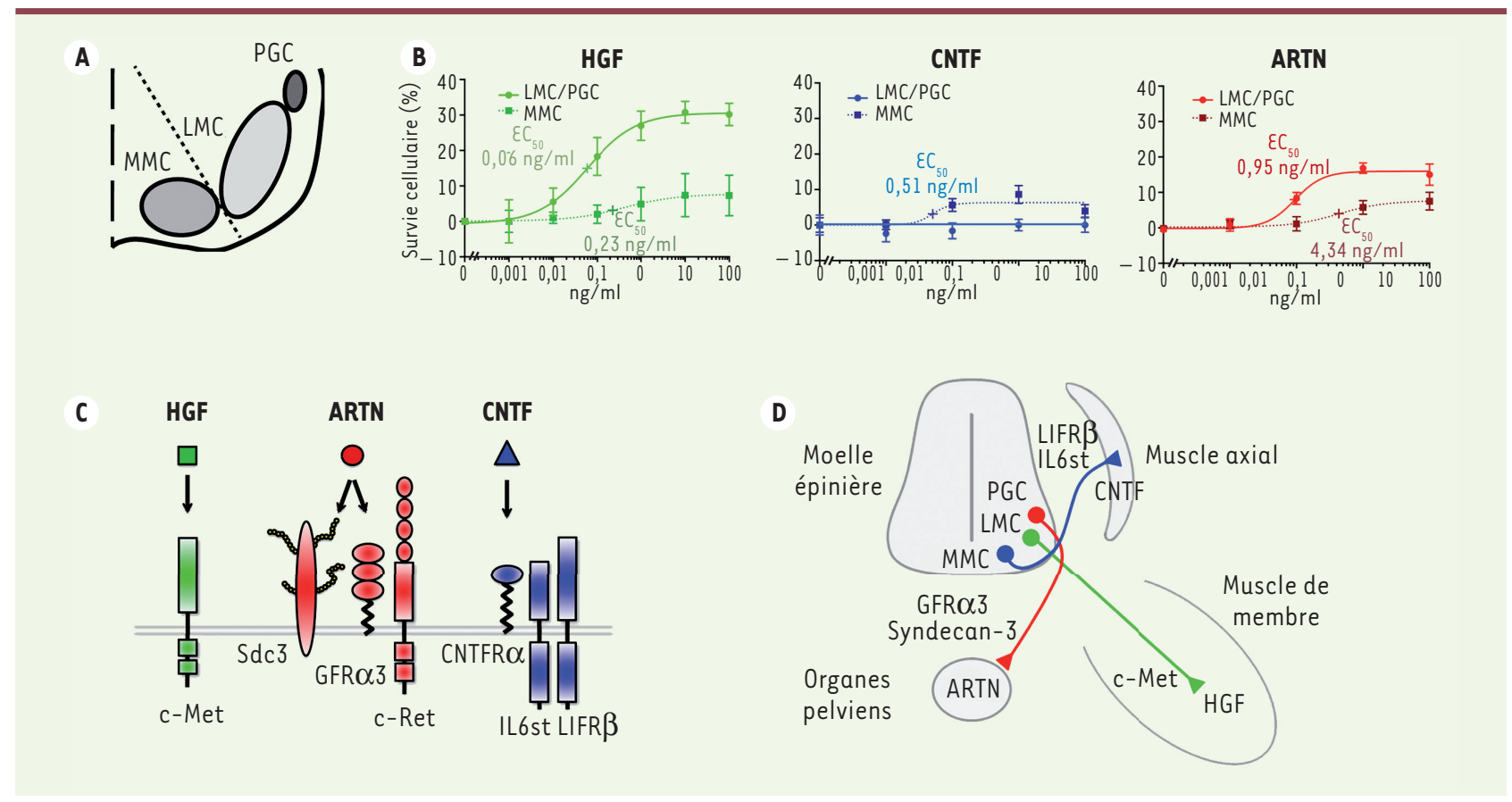

Figure 2. Sous-populations de motoneurones répondant aux facteurs HGF, CNTF et ARTN. A. Schématisation des trois principales sous-populations de motoneurones de la moelle épinière lombaire : les motoneurones des colonnes motrices médiane (MMC) et latérale (LMC) et les neurones préganglionnaires $(P G C)$. Les motoneurones MMC sont isolés des populations LMC et PGC par dissection anatomique (ligne pointillée), elle-même suivie par un tri cellulaire. B. Courbes de survie après trois jours de traitement in vitro. Les facteurs HGF (hepatocyte growth factor) et artémine (ARTN) favorisent préférentiellement la survie des motoneurones LMC/PGC. Le CNTF (ciliary neurotrophic factor) permet de manière sélective la survie de motoneurones MMC. C. Schématisation des récepteurs pour les facteurs neurotrophiques HGF (carré vert), ARTN (rond rouge) et CNTF (triangle bleu). Le HGF se lie au récepteur tyrosine kinase c-Met. L'ARTN se lie à un récepteur multimérique composé de la tyrosine kinase c-Ret, de GRF 33 (GDNF family receptor $\alpha 3$ ), et du protéoglycane Sdc3 (syndecan-3). Le CNTF se lie à un récepteur multimérique composé de CNTFR $\alpha$ (ciliary neurotrophic factor receptor $\alpha$ ), IL6st (interleukin 6 signal transducer) et LIFR $\beta$ (LIF receptor $\beta$ ). D. Schématisation des sous-populations de motoneurones LMC, MMC et PGC et des signalisations permettant leur survie.

accord avec son expression restreinte dans les muscles des membres [7]. Le facteur CNTF agit spécifiquement sur les motoneurones de la colonne motrice médiane au stade précoce de développement analysé dans cette étude. Enfin, I'ARTN apparaît comme un nouveau facteur de survie pour les motoneurones préganglionnaires du système parasympathique qui innervent les neurones postganglionnaires du côlon distal, de la vessie et des organes génitaux. Ceci est en concordance avec l'expression d'ARTN au niveau de ces organes [8], et également avec celle de son récepteur GFR $\alpha 3$ dans les corps cellulaires et les axones des motoneurones préganglionnaires.

Ces observations ont-elles des implications thérapeutiques? Dans des modèles de sclérose latérale amyotrophique
(SLA) et d'amyotrophie spinale infantile (SMA), plusieurs facteurs neurotrophiques ont montré in vivo un bénéfice thérapeutique. En effet, dans un modèle murin de SLA, des souris transgéniques qui expriment des formes mutées de SODl (superoxyde

dismutase - 1$)[8, \quad(\rightarrow)$ Voir la Nouvelle de 9] B. Pettmann et al., $\mathrm{m} / \mathrm{s}$ 9] $(\rightarrow)$, quatre $n^{\circ} 11$, novembre 2006, facteurs neuro- page 923 trophiques se sont

avérés être parmi les six traitements les plus efficaces testés (à l'exception, comme attendu, des modificateurs de SOD1) [10]. Nos données sur l'action préférentielle de I'HGF vis-à-vis des motoneurones LMC pourraient expliquer les puissants effets thérapeutiques de ce facteur contre la fonte de la masse musculaire et la parésie des membres, chez les souris [11]. Cependant, pour la plupart des facteurs neurotrophiques, les motoneurones qui en sont la cible restent à identifier aux stades adultes. Des études sont actuellement en cours afin de déterminer les besoins trophiques des motoneurones les plus vulnérables dans diverses maladies dégénératives: les motoneurones des membres dans la SLA classique, ceux proximaux dans la SMA, ou encore bulbaires dans certaines formes de SLA ou d'amyotrophie spinobulbaire (SBMA). $\diamond$

Neurotrophic factors for motor neurons: an à la carte menu

\section{LIENS D'INTÉRÊT}

Les auteurs déclarent n'avoir aucun lien d'intérêt concernant les données publiées dans cet article. 


\section{RÉFÉRENCES}

1. Dale Purves. Body and brain, a trophic theory of neural development. Cambridge, Mass : Harvard University Press, 1986.

2. Gould TW, Enomoto H. Neurotrophic modulation of motor neuron development. Neuroscientist 2009; 15 : 105-16.

3. Kanning KC, Kaplan A, Henderson CE. Motor neuron diversity in development and disease. Annu Rev Neurosci $2010 ; 33: 409-40$.

4. Haase G, Kennel P, Pettmann B, et al. Gene therapy of murine motor neuron disease using adenoviral vectors for neurotrophic factors. Nat Med 1997 ; 3 : 429-36.

5. Haase G, Dessaud $\varepsilon$, Garces A, et al. Gdnf acts through Pea3 to regulate cell body positioning and muscle innervation of specific motor neuron pools. Neuron $2002 ; 35: 893-905$

6. Schaller S, Buttigieg D, Alory A, et al. Novel combinatorial screening identifies neurotrophic factors for selective classes of motor neurons. Proc Natl Acad Sci USA 2017 ; 114 : ع2486-93.

7. Sonnenberg $\varepsilon$, Meyer D, Weidner KM, et al. Scatter factor/hepatocyte growth factor and its receptor, the c-met tyrosine kinase, can mediate a signal exchange between mesenchyme and epithelia during mouse development. J Cell Biol 1993; 123 : 223-35.

8. Bolon B, Jing S, Asuncion F, et al. The candidate neuroprotective agent artemin induces autonomic neural dysplasia without preventing peripheral nerve dysfunction. Toxicol Pathol 2004 ; 32 : 275-94.

\section{NOUVELLE}

\section{Quand le cytosquelette d'actine fait le jeu des rétrovirus}

Élodie Teruel ${ }^{\star}$, Gerges Rizkallah $^{\star}$, Chloé Journo $^{\star \star}$, Hélène Dutartre ${ }^{\star \star}$
Le rétrovirus $\mathrm{HTLV}-1$, un modèle de choix pour étudier le rôle du cytosquelette dans la transmission virale

Les virus, «parasites » intracellulaires obligatoires, exploitent diverses stratégies pour se propager au sein de I'hôte qu'ils infectent. Les particules virales peuvent être libérées dans le milieu extracellulaire ou profiter des contacts entre cellules pour se propager directement, de cellule à cellule, en évitant l'environnement cellulaire. Ces contacts peuvent prendre la forme d'extensions (comme les filopodes ou les nanotubes) ou reposer sur une zone de contact étendue appelée «synapse virologique » (SV). La formation de cette synapse nécessite l'établissement d'une zone d'adhérence entre les cellules et l'adressage des éléments viraux de la cellule infectée vers cette zone de contact avec la cellule saine, deux processus qui reposent sur des remaniements du cytosquelette au sein de la cellule infectée. Le rétrovirus HTLV-1 (human T-lymphotropic virus type 1) se transmet principalement par contact cellulaire, les par-

ticules virales

$(\rightarrow)$ Voir la Synthèse de G. Rizkallah et al., libres n'étant que $m / s n^{\circ} 6-7$, juin-juillet peu infectieuses 2015, page 629 [1] $(\rightarrow)$.

Tax, la protéine transactivatrice du virus HTLV-1, induit l'expression de Gem, une protéine membre des petites protéines fixant le GTP de la famille de la superfamille Ras, qui est associée au cytosquelette d'actine et contribue à la migration cellulaire et à la formation de zones de contact (ou conjugués) entre les cellules infectées et les cellules cibles [2]. La protéine Tax induit également l'expression de la
9. Pettmann B, Raoul C, Haase G. Mort des motoneuronrs dans la SLA : suicide ou meurtre? Med Sci (Paris) $2006 ; 22$ : 923-5.

10. Turner BJ, Talbot K. Transgenics, toxicity and therapeutics in rodent models of mutant sodlmediated familial als. Prog Neurobiol 2008 ; 85 : 94-134.

11. Sun W, Funakoshi H, Nakamura T. Overexpression of hgf retards disease progression and prolongs life span in a transgenic mouse model of als. J Neurosci 2002 ; $22: 6537-48$
Centre international de recherche en infec-

tiologie, équipe oncogenèse rétrovirale, Inserm U1111 - université Claude Bernard Lyon 1, CNRS, UMR5308, École normale supérieure de Lyon, université de Lyon, 46, allée d'Italie, F-69007, Lyon, France ; équipe labellisée « Ligue nationale contre le cancer».

* Contribution égale en tant que premiers auteurs.

${ }^{* *}$ Contribution égale en tant qu'auteurs seniors.

helene.dutartre@ens-lyon.fr chloe.journo@ens-lyon.fr

protéine d'adhérence ICAM-1 (intercellular adhesion molecule 1) qui stabilise les contacts entre cellules. La signalisation induite à la suite de la polarisation d'ICAM-1 à la surface de la cellule infectée, conduit au positionnement du centre organisateur des microtubules vers la synapse virologique, ce qui favoriserait la transmission virale et le ciblage vers les cellules saines [3]. La fascine contrôle la formation des faisceaux d'actine à partir de l'actine filamenteuse [4]. Elle favorise la migration et l'adhérence cellulaires [5], et stabilise les protrusions et les filopodes, ce qui facilite les contacts entre les cellules $[6,7]$. Récemment, il a été montré que l'expression de la fascine était induite par $\operatorname{Tax}[8,9]$ et que la protéine participait à la transmission du virus [10]. 